\title{
The meaning of MSCT - angiography in early detection of visceral arterial lesions in patients with antiphospholipid syndrome
}

\section{Authors:}

Jovica Saponjski,

Ljudmila Stojanovich,

Jelena Petrovic,

Dusan Saponjski*

* Corresponding author.

* Prof.dr Jovica Saponjski, MD, PhD

E-mail address: sapica1961@yahoo.com

Professor of Radiology. Institute of cardio-vascular diseases, Clinical Center of Serbia, Belgrade, Serbia.

* Ljudmila Stojanovich, MD, PhD

Internal medicine, "Bezanijska Kosa", University Medical Center, Belgrade

E-Mail:

ljudmila_stojanovich@yahoo.com

* Jelena Petrovic, MD

School of medicine, University of

Belgrade.

E-Mail: jelena_petrovic18@yahoo.com

* Dusan Saponski, MD,

* Corresponding author

Center for radiology and MR, Clinical

Center of Serbia, Belgrade, Serbia.

E-Mail: saponjski.d@gmail.com,

tel.: +38169 22-92-887

Key words: antiphospholipid syndrome, vascular manifestations, 64-multi sliced computed tomography

\begin{abstract}
:
Introduction: Antiphospholipid syndrome (APS) is an autoimmune disease which is characterized by arterial and venous thrombosis, fetal loss, and the presence of antiphospholipid antibodies in the serum (aPL). It is characterized by accelerated atherosclerosis and that together with an increased tendency towards thrombosis leading to the occurrence of various vascular events.
\end{abstract}

The aim of the study: The goal of our work is to show the results we obtained in MSCT angiography examination of visceral arteries included patients with primary APS (PAPS) and secondary APS (SAPS), comparing them with a control group.

Methods: In this study we analyzed 50 patients with primary antiphospholipid syndrome (PAPS) and 50 patients, who have secondary antiphospholipid syndrome (SAPS). The results were compared to 50 controls. The groups were comparable with respect to age, gender, and traditional risk factors except for the lipid status, since controls had significantly higher levels of cholesterol and triglycerides. The study was conducted on 64multi-sliced computed tomography (MSCT), where we analyzed the quantitative and morphological characteristics of blood vessel lesions.

Results: Patients from the control group had statistically significant elevated cholesterol and triglyceride levels compared to the patients with SAPS and PAPS $(p<0.001$ and $p<0.05)$. The results showed that the frequency change is statistically ( $p<0.05$ and $p<0.001)$ more frequent in patients with APS than in the control group. Analyzing the percentage of diameter stenosis, we have established that lesions from the group with 0\%-30\% diameter stenosis (DS) and 30\%-50\% DS are in patients with PAPS $(n=48)$ and SAPS $(n=42)$ are more common than in the control group $(n=20, p<0.05)$. Analyzing the qualitative characteristics of plaques we have established a significantly higher frequency of soft tissue $(n=21)$ and mixed lesions $(n=25)$ in patients with PAPS than the calcified one $(\mathrm{n}=2, \mathrm{p}<0.001)$.

Conclusion: Our study showed that the subclinical manifestation of changes on visceral arteries is more common in patients with APS. Because of its safety and accuracy, the method of choice is MSCT angiography in monitoring disease progression. 


\section{Introduction}

Antiphospholipid syndrome (APS) is an autoimmune disease which is characterized by arterial and venous thrombosis, fetal loss, and the presence of antiphospholipid antibodies in the serum (aPL). APS can be primary - as the primary disease (PAPS), secondary within some other autoimmune disease SAPS, and the worst form with a very high mortality rate - catastrophic APS [1] Preliminary criteria (clinical and laboratory) for APS classification were defined in 1999 by Sapporo et al., and updated in 2006 in Sydney et al. [2,3,4]. Laboratory criteria for diagnosing the disease include the presence of lupus anticoagulant (LA) in blood, a protein that increases the tendency of thrombosis (thrombophilia) in arteries and veins, and anticardiolipin antibodies (aCL) of $\mathrm{IgG}$ or IgM class. Those antibodies should be in moderate or high titer, detected two or more times with at least six weeks interval [6]. It has been noted that these patients have elevated reactive function of the hematopoietic system, with increased tendency of thrombosis (thrombophilia) in arteries and veins. $[5,6,7,8]$
It is known that one of the most common APS manifestations are sudden blood vessel occlusions (thrombosis) and premature atherosclerosis, with thrombosis in head and neck blood vessels, visceral organ blood vessels, as well as in the lower extremities' ones. Therefore, early diagnosis of blood vessel changes is crucial in these patients, and important in planning the treatment approach. Until recently, color Doppler ultrasound (Doppler) examination of blood vessels was the only non-invasive method used to diagnose changes within blood vessel lumen. Doppler obtained good results in the review of major blood vessels such as the carotid arteries, as well as the arteries of the upper and lower extremities. Now 64-sliced computed tomography (MSCT), as a non-invasive method, is used in everyday diagnostics of occlusive blood vessel changes. It allows excellent visualization of all major and minor blood vessels, their morphology, blood vessels interior (virtual angiography), and plaque morphology $[11-13,26]$.

The aim of this study was to compare incidence of lesion severity and 
their morphological characteristics in patients with APS (PAPS and SAPS) and the control group.

\section{Methods}

In this case-control study, we enrolled 50 PAPS patients (mean age $53 \pm 11$ years, 29 female, 21 men), 50 SAPS patients $(53 \pm 9$ years, 31 female, 19 men), and 50 control patients without established autoimmune disease $(54 \pm 8$ years, 14 female, 36 men); Analyzing that data, difference in the age of patients was not statistically significant $(p>0.05)$, while for gender was statistically significant $(\mathrm{p}<0.05)$. The main indication for MSCT angiography was evaluation of APS in patients with PAPS and SAPS, while the control group consisted of patients without APS who were indicated for MSCT angiography because of peripheral vascular disease. Exclusion criteria were pregnancy or breast-feeding, severe impairment of respiratory, cardiac or renal function and fever.

Diagnosis of APS was made according to Sydney APS classification criteria (2006). Besides clinical symptoms and signs of APS, we also tested patients' blood for antiphospholipid antibodies (aPL). Laboratory diagnostics determined the results of the phospholipid-dependent coagulation tests for detection of lupus anticoagulants (LA) and immunofluorescence (ELISA) tests for evidence of anticardiolipin antibodies (aCL), IgG and IgM class, as well as AntiB2 glycoprotein-I antibody of IgG and/or IgM isotype in serum or plasma[6].

In all patients, standard laboratory analysis and evaluation of atherosclerosis risk factors (dyslipidemia, hyperglycemia, smoking habits, hypertension, and family history) were performed. After vascular surgeon`s exam, Doppler ultrasound of visceral organ blood vessels was performed. Following these procedures, MSCT angiography was also indicated.

MSCT angiographic examination was pointed to visceral arteries (abdominal aorta, truncus coeliacus, artery mesenterica superior, artery mesenterica inferior and both renal arteries) with whole-body angiography (WBA) time 26 sec - which means, less than a minute, with application of 70 up to $120 \mathrm{ml}$ of contrast agent (Ioversol 350) followed by $30 \mathrm{ml}$ of physiological solution. Results 
were analyzed by an integrated system of quantitative peripheral angiography, giving the data of stenosis diameter. Changes of blood vessels which were analyzed in patients with subclinical form had not existed before our MSCT examination.

The changes we found on the visceral arteries have been classified in four groups based on the percentage of diameter stenosis (DS): 0\%-30\% DS; $31 \%-50 \% ; 51 \%-70 \%$ and $>71 \%$. All of the blood vessel lesions have been divided in three groups based on morphological characteristics: soft tissue lesions, calcified lesions and mixed type lesions.

For statistical analysis, ANOVA and Tukey's range test have been used.

\section{Results}

Regarding risk factors, patients from the control group had statistically significantly higher cholesterol levels in comparison to the patients with SAPS and PAPS $(p<0.001)$. Also, the patients from the control group had significantly higher triglyceride levels in comparison to SAPS and PAPS patients $(\mathrm{p}<0.05)$. Compared to other analyzed risk factors, no significant statistical difference between the groups was found (Table 1).

By MSCT angiography, there was a higher frequency of the changes on the arteries in the patients with APS. In patients with PAPS, as well as in SAPS there were significantly more atherosclerotic changes than in the control group (48 patients with PAPS vs 20 patients in the control group) $\mathrm{p}<0.0 .5$ and (42 pt. with SAPS vs 20 pt. in control group), $\mathrm{p}<0.05$.

Analyzing the percentage of diameter stenosis (DS), we have found that the lesions from the group with $0 \%$ $30 \%$ DS in patients with PAPS (32/48) and SAPS (26/42) were more common than in the control group (9/27). The frequency of lesions in the group with $30 \%-50 \%$ DS has not been statistically significant between patients with APS and control group (13/48 vs 12/42, vs 7/27). The number of patients with 50\%-70\% DS of visceral organ blood vessels was more frequent in the control group $(n=8 / 27)$, than in the patients with PAPS $(n=3 / 48$, $\mathrm{p}<0.05$ ), as well as in the patients with SAPS $(n=4 / 42, p<0.05)$. It is interesting to note that the incidence of lesions higher 
than $70 \%$ DS in the control group $(3 / 27$, $75 \%$ ) was more statistically significant than in the patients with SAPS (0/42, $\mathrm{p}<0.001)$, and in the patients with PAPS $(1 / 48, \mathrm{p}<0.001)$.

Analyzing the qualitative characteristics of plaques, we divided all lesions in 3 groups: soft tissue plaques with minimum calcium in the plaques, then the mixed ones with calcium and other cell elements in similar amount in the plaques, and the calcified ones, the content of which largely makes calcium. We have established a significantly higher frequency of soft tissue (24/48) and mixed lesions $(21 / 48,48.0 \%)$ in patients with PAPS than the calcified ones (3/48, $\mathrm{p}<0.001)$. In patients with SAPS, there were significantly more mixed lesions (22/42) $(\mathrm{p}<0.001)$, and significantly more soft tissue lesions (16/42) than calcified plaques $(4 / 42,18.37 \%),(\mathrm{p}<0.05)$. On the other hand, in the control group there were mainly calcified lesions $(16 / 27, \mathrm{p}<0.001)$ in comparison to soft tissue plaques $(3 / 27$, $18.37 \%)$ and mixed tissue plaques (8/27, $\mathrm{p}<0.05)$.

\section{Discussion}

In this study, that included 100 patients with APS (50 with PAPS and 50 with SAPS), we analyzed the frequency and morphological characteristics of the arterial lesions of visceral organs that were detected during MSCT-angiography. We were especially interested in the role of this non-invasive method in the early detection of subclinical manifestations in patients with APS. By comparing the results and analyzing the role of risk factors on the course of the disease, we noticed that some values of the risk factors were significantly higher in the control group. This implies that the classic risk factors are not of key importance for accelerated development of atherosclerosis and changes in the blood vessels in patients with APS. Normally, any occurrence of risk factors elevated values in patients with APS must be treated, and biochemical values must be brought into the normal parameters.

MSCT angiography has given us a lot of data that could not be obtained so accurately by any other diagnostic method. [14,15] From the obtained results we can conclude that the occurrence of 
blood vessel lesions in patients with APS is far more frequent than in the control group. MSCT angiography was found to be the method of choice for monitoring the course of the disease, and for the early detection of changes in the blood vessels $[16,17]$. Our results have shown that visceral arteries lesions are statistically more often found in patients with APS. The largest number of lesions in patients with APS have a percentage of diameter stenosis less than $70 \%$ and those lesions are in soft tissues and mixed lesions group. By comparing the obtained results with other results from the literature, we have demonstrated that this method is superior to the Doppler ultrasonographic examination because of its ability to visualize the lesions that are less than $40 \%$ lumen narrowed, as well as smaller lumen blood vessels. [18,19,20]

Classic angiography, which has been the "gold standard" in diagnostics, as an invasive diagnostics is slowly losing its importance, and MSCT angiography is taking its place. When we compare these two methods, MSCT angiography has the advantage - mostly because it is a non-invasive method, with a minimum risk. [21,22]

Doppler would be an ideal first diagnostic option, but not all visceral organ blood vessels are approachable for examination in all the patients, and therefore it couldn't be the first method of choice. In our study, Doppler was performed on all the patients, but the results were not satisfying and did not have statistic relevance.

Magnetic resonance angiography (MRA) is for the purposes of daily usage still under development, but it will find its place in the future mostly as a noninvasive method without any X-ray radiation. The downfalls are that the spatial resolution of MRA is still inferior to conventional angiographies, the exam is more expensive and lasts longer than the MSCT's one. The full realization of the clinical potential of MRA requires continuing clinical studies and further improvements with regard to the efficiency of the technique, spatial and contrast resolution, suppression of artifacts, processing and display [23-25].

At the end, the conclusion is: MSCT is a vascular imaging technique based on 
the rapid acquisition of volumetric MSCT data during the bolus phase of intravenously administered contrast media. If we consider the fact that there are virtually no complications, except allergic reactions to contrast, we can consider it as the low-risk method. With MSCT angiography, the possibility of interpretation error has been ruled out, unlike the ultrasound examination of blood vessels. MSCT can allow us to visualize the changes on all blood vessels in one pass, which is of special importance for evaluation of the course and treatment of patients with APS.

\section{Acknowledgements:}

We thank all patients who participated in this study and our colleagues from many clinics in Serbia. This work was supported by research grant number 175041, issued by the Ministry of Science of the Republic of Serbia.

\section{Conflicts of interest:}

The authors declare no conflicts of interest. 


\section{References}

1. Lockshin MD, Sammartino LR, Schwartzman S. Validation of the Sapporo criteria for antiphospholipid syndrome. Arthritis Rheum 2000;43:440-443.

2. Miyakis S, Lockshin MD, Atsumi T, Branch DW, Brey RL, Cervera R, Derksen RH, DE Groot PG, Koike T, Meroni PL, Reber G, Shoenfeld Y, Tincani A, Vlachoyiannopoulos PG, Krilis SA (February 2006). "International consensus statement on an update of the classification criteria for definite antiphospholipid syndrome (APS)". J. Thromb. Haemost.4 (2): 295-306.

3. Shoenfeld Y, Sherer Y, Haratz D. Atherosclerosis as an infectious, inflammatory and autoimmune disease. Trends Immunol 2001;22:293-5.

4. Ames PR, Margarita A, Sokoll KB, Weston M, Brancaccio V. Premature atherosclerosis in primary antiphospholipid syndrome: preliminary data. Ann Rheum Dis 2005;64:315-317.
5. Rovensky J. Asheron's syndrome catastrophic antipospholipid syndrome. Autoimmun Rev 2006;6:61-3.

6. Ruiz-Irastorza G, Crowther M, Branch W, Khamashta MA. Antiphospholipid syndrome. Lancet 2010; 376:1498.

7. Shoenfeld Y, Zandman-Goddard G, Stojanovich L, et al. The mosaic of autoimmunity: hormonal and environmental factors involved in autoimmune diseases - 2008. Isr Med Assoc J 2008;10(1):8-12.

8. Shoenfeld Y, Gerli R, Doria A, et al. Accelerated Atherosclerosis in Autoimmune Rheumatic Diseases. Circulation. 2005;112:3337-3347.

9. Krause I, Blank M, Stojanovich L, et al: The association of thrombocytopenia with systemic manifestations in the antiphospholipid syndrome. Immunobiology 2005; 210:749-754.

10. Jiménez S, García-Criado MA, Tàssies $\mathrm{D}$, et al. Preclinical vascular disease in systemic lupus erythematosus and 
primary antiphospholipid syndrome. Rheumatology 2005;44:756-761

11. Soltész P, Szekanecz Z, Kiss E, Shoenfeld Y. Cardiac manifestations in antiphospholipid syndrome. Autoimmun Rev. 2007;6(6):379-86.

12. Saponjski J, Stojanovich L, Djokovic A, Petkovic M, Mrda D.: Systemic Vascular Diseases in the Antiphospholipid Syndrome. What is the best diagnostic choice? Autoimmun Rev. 2010 Nov 21.

13. Lalani TA, Kanne JP, Hatfield GA, Chen P. Imaging findings in systemic lupus erythematosus. Radiographics. 2004;24(4):1069-86.

14. Kaushik S, Federle M, Schur PH, Krishnan M, Silverman SG, Ros PR. Abdominal thrombotic and ischemic manifestations of the antiphospholipid antibody syndrome: CT findings in 42 patients. Radiology 2001;218:768-7.

15. Beregi JP, Djabbari M, Desmoucelle F, Willoteaux S, Wattinne L, Louvegini S. Popliteal vascular disease: evaluation with spiral CT angiography. Radiology 1997;203:477-483.
16. Verbeeck N, Cavez N, Plawny L, Weber J, Lens V. Primary aortic thrombosis: role of enhanced multislice CT demonstrated in three exceptional cases. JBR-BTR. 2014 Mar-Apr; 97(2):76-80.

17. Suzuki K, Uemura T, Kikuchi M, Ishihara Y, Ichioka S. Acute LimbThreatening Ischemia Associated With Antiphospholipid Syndrome: A Report of Two Cases. J Foot Ankle Surg. 2016 Feb 19.

18. Crome CR, Rajagopalan S, Kuhan G, Fluck N. Antiphospholipid syndrome presenting with acute digital ischaemia, avascular necrosis of the femoral head and superior mesenteric artery thrombus. BMJ Case Rep. 2012 Nov $20 ; 2012$.

19. Sitia S, Atzeni F, Sarzi-Puttini P, Di Bello V, Tomasoni L, Delfino L, Antonini-Canterin F, Di Salvo G, De Gennaro Colonna V, La Carrubba S, Carerj S,Turiel M. Cardiovascular involvement in systemic autoimmune diseases. Autoimmun Rev. 2009 Feb;8(4):281-6. 
20. Langenberger $H$, Schillinger M, Plank C, Sabeti S, Dick P, Cejna M, Lammer J,Minar E, Loewe C. Agreement of duplex ultrasonography vs computed tomography angiography for evaluation of native and in-stent SFA re-stenosis-findings from a randomized controlled trial. Eur J Radiol. 2012 Sep;81(9):2265-9.

21. Laswed T, Rizzo E, Guntern D, Doenz F, Denys A, Schnyder P, Qanadli SD.Assessment of occlusive arterial disease of abdominal aorta and lower extremities arteries: value of multidetector CT angiography using an adaptive acquisition method. Eur Radiol. 2008 Feb;18(2):263-72.

22. Nardi P, Pellegrino A, Romagnoli A, Mve Mvondo C, De Propris S, Sperandio M,Versaci F, Simonetti G, Chiariello L. Multidetector computed tomographic coronary angiography as an alternative to conventional coronary angiography in non-coronary surgical patients. J Cardiovasc Surg (Torino). 2011 Jun;52(3):429-35.

23. Van den Bosch H, Westenberg J, SetzPels W, Kersten E, Tielbeek A, Duijm
L,Post J, Teijink J, de Roos A. Prognostic value of cardiovascular MR imaging biomarkers on outcome in peripheral arterial disease: a 6-year follow-up pilot study. Int J Cardiovasc Imaging. 2016 May 21.

24. Lin $\mathrm{YH}$, Lin $\mathrm{HH}$, Liu HM, Lee CW, Chen YF. Diagnostic performance of CT and MRI on the detection of symptomatic intracranial dural arteriovenous fistula: a meta-analysis with indirect comparison. Neuroradiology. 2016 May 16.

25. Cina A, Di Stasi C, Semeraro V, Marano R, Savino G, Iezzi R, Bonomo L.Comparison of CT and MR angiography in evaluation of peripheral arterial disease before endovascular intervention. Acta Radiol. 2016 May; 57(5):547-56.

26. Jimenez S, Garcia-Criado A, Tassies D, et al., Preclinical vascular disease in systemic lupus erythematosus and primary antiphospholipid syndrome, Rheumatology 2005; 44: 756-761. 
Table 1: Baseline study population characteristics

\begin{tabular}{l|llll}
\hline Risk factors & PAPS & SAPS & Controls & $\mathrm{p}$ \\
\hline Age (years) & $52.98 \pm 11.24$ & $53.26 \pm 9.29$ & $54.47 \pm 8.04$ & $\mathrm{p} \geq 0.05$ \\
Cholesterol levels (mmol/l) & $4.86 \pm 1.04$ & $4.85 \pm 0.86$ & $5.94 \pm 0.57$ & $\mathrm{p}<0.001$ \\
Triglyceride levels (mmol/l) & $1.33 \pm 0.49$ & $1.36 \pm 0.48$ & $1.71 \pm 0.56$ & $\mathrm{p}<0.05$ \\
\hline Glycaemia levels (mmol/l) & $5.05 \pm 1.04$ & $4.92 \pm 0.69$ & $5.09 \pm 0.85$ & $\mathrm{p} \geq 0.05$ \\
\hline
\end{tabular}

